\title{
Automatic versus User-Controlled Methods of Briefly Interrupting Telephone Calls
}

\author{
ROBERT B. KATZ,' Eatontown, New Jersey
}

\begin{abstract}
Some future telephone services will require that ongoing calls be interrupted briefly so that one of the parties can receive data transmitted over the voice path. For example, the new service Caller ID on Call Waiting (CIDCW) will allow subscribers who are off-hook and engaged in conversation to receive data indicating the name and telephone number of a new caller, but the data transmission will produce a break of approximately $1 \mathrm{~s}$. The question arises, then, as to what method should be used to interrupt calls to transmit data. In an automatic form of CIDCW, data would be sent without user control once there is a new call, producing an unexpected break in ongoing conversations. In a user-controlled form, users would hear a tone when there is a new call and initiate the data transmission by pressing a button. A study was conducted to examine how subjects would react to breaks in their telephone conversations and to determine which form of CIDCW was more favorable. Subjects acting as CIDCW subscribers liked both forms of the service, but they preferred the automatic form, which was also rated as more acceptable and easier to use. Furthermore, compared with the user-controlled form, automatic CIDCW resulted in quick, error-free performance. Subjects acting as the far-end party also favored the automatic form, provided that they heard silence during the interruptions rather than a tone. Generally the automatic approach was found to be the better way to introduce a 1-s break into telephone calls.
\end{abstract}

\section{INTRODUCTION}

Some future telephone services will require that ongoing telephone calls be interrupted briefly so that one of the parties can receive data transmitted over the voice path. A service like this is called Caller ID on Call Waiting (CIDCW). CIDCW is a new service that integrates Call Waiting and two services that provide information about an incoming call, Calling Number Delivery and Calling Name Delivery. Call Waiting allows customers who are having a telephone conversation to know that someone is calling them-customers hear a short tone when there is a new call. Calling Number Delivery and

\footnotetext{
${ }^{1}$ Requests for reprints should be sent to Robert B. Katz, 31 Victoria Drive, Eatontown, NJ 07724, rbkatz@aol.com.
}

Calling Name Delivery (often collectively referred to as Caller ID) provide the telephone number and name of a caller to customers whose telephones are on-hook-the caller ID information appears on a display screen attached to the telephone.

Currently, customers cannot receive information about the identity of a new caller while they are having a telephone conversation. The purpose of CIDCW is to remedy this situation by allowing customers who are off-hook and engaged in conversation to receive the directory number and name of new callers.

A key problem in the deployment of CIDCW is how to transmit caller ID data to a customer's premises over the voice path while the voice path is being used for conversation. Transmission 
of data during conversation would not be a problem in, for example, Integrated Services Digital Network (Bellcore, 1989), which provides for a data channel that is separate from the voice path. Without a separate data channel, perhaps the most elegant solution would be to use a technique for sending data and voice over the same channel simultaneously (e.g., Schilling, Pickholtz, and Milstein, 1990).

However, use of such a technique could entail relatively high costs (for customers' telephone equipment and for telephone companies' modifications to their switching systems) and long deployment times. Therefore, it was decided that caller ID information should be transmitted via frequency shift keying during telephone calls. Thus telephone conversations will have to be interrupted briefly (approximately $1 \mathrm{~s}$ ) so that the data can be transmitted over a clear channel. The question arises, then, as to what method should be used to interrupt a call in order to provide the clear data channel.

Two methods of implementing CIDCW may be considered: an automatic method and a usercontrolled method. Both have the goal of transmitting caller ID data over the voice path-after the voice path has already been put into use for telephone conversation-in such a way that speech and background noise do not corrupt the data and that customers do not hear the data transmission. In automatic CIDCW, as soon as there is a new call, the telephone company switch silences the voice path of the far-end party, preventing that party's speech from interfering with the data transmission. The switch then sends a signal to the CIDCW subscriber's customer premises equipment (CPE), which should mute the telephone handset, preventing the subscriber's speech from corrupting the data and keeping the subscriber from hearing the data transmission. The signal also alerts the subscriber to the presence of the new call. At this point, the switch sends the data, then restores the far-end connection, and the CPE unmutes the subscriber's handset. This form of CIDCW is referred to as automatic because subscribers do not have to take any action to receive caller ID data; the data are sent automatically.

In user-controlled CIDCW, subscribers initiate transmission of the data. When there is a new call, the switch alerts the subscriber by sending a Call Waiting tone, during which time the farend party's connection is silenced briefly. The subscriber then presses a button on the CPE to initiate the data transmission sequence. As before, the subscriber's CPE mutes the handset, and the switch, upon receiving the signal from the CPE, silences the voice path of the far-end party and sends the data. (Both forms of CIDCW would require a new CPE capable of muting the handset. The user-controlled form may additionally need a special button to initiate the data transmission.)

From a human factors standpoint, there are advantages and disadvantages associated with both automatic and user-controlled CIDCW. The major advantage of automatic CIDCW is that subscribers need take no action to receive caller ID data; they do not have to learn any new responses or the use of new buttons. This also means that subscribers do not have to break their conversations, excusing themselves from the other party, to find out who is calling. In fact, from the subscribers' perspective, automatic CIDCW is similar to Call Waiting service except there is an additional interval following the tone during which caller ID data are sent and communication is not possible. Considering that subscribers do not have to decide whether or when to receive caller ID data, they may be able to answer a new call quickly when they want to do so.

Automatic CIDCW has a major disadvantage, however. Because the switch sends the data once a new call arrives, ongoing conversations can be interrupted at any time without warning. Subscribers would be aware that an interruption might occur, but they would not know when it would occur. The far-end party would not necessarily know even that there might be an interruption. The high degree of redundancy in language may mean that short gaps in 
conversation usually would not lead to any loss of information (e.g., Miller and Licklider, 1950). However, a gap of $1 \mathrm{~s}$ could be long enough for one or two crucial words not to be conveyed if the conversation continued through a CIDCW interruption. Considering that the CIDCW subscriber would probably shift attention from the conversation to the Caller ID display to see who was calling, the interruption could disrupt the conversation more than would be expected by a 1-s gap.

User-controlled CIDCW does not have the disadvantage of automatic CIDCW. Because subscribers decide when caller ID data are transmitted, disruption to ongoing conversations could be minimized. User-controlled CIDCW is also more flexible than the automatic form. In the automatic form, the switch tries to send caller ID data for every call. In the usercontrolled form, receiving the data is not a mandatory step; subscribers have the option of answering the new call without first finding out who is calling.

User-controlled CIDCW has three disadvantages: (1) subscribers may have to learn to use a special button on the CPE to initiate the data transmission; (2) subscribers might introduce a formal break into their conversations to find out who is calling, and this break might be disruptive; and (3) subscribers might delay the data transmission so long that, by the time they decide to be connected with the new caller, the new caller may sometimes have hung up or the call may have been forwarded to a voice mail system.

The present study was conducted, first, to examine how customers would react to interruptions to their telephone calls for purposes of receiving data and, second, to determine which form would be preferred: automatic or usercontrolled CIDCW. Although prior studies have examined customer reactions to various forms of telephone network impairment-such as echo, loss, and noise (e.g., Cavanaugh, Hatch, and Sullivan, 1976), gaps and clipping (e.g., Gruber and Strawczynski, 1985), and delay (e.g.,
Klemmer, 1967)-none had looked at interruptions like those imposed by CIDCW.

Accordingly, pairs of subjects communicating over the telephone were tested in the laboratory. One subject played the role of the CIDCW subscriber and the other played the far-end party. The subjects were tested in a situation that simulated the interruptions of automatic CIDCW and of user-controlled CIDCW. Both the subscribers and the far-end subjects filled out questionnaires to assess their preferences. The study also examined whether user preference varied with prior experience with Caller ID service or Call Waiting service and whether it depended on which subject (subscriber or far-end party) was talking when the interruptions occurred. The latter was included as an experimental manipulation because CIDCW interruptions might be considered more unacceptable by subjects if they occurred when the other party was talking, given that gaps in the other party's discourse could be apparent. Indeed, pilot work demonstrated that subjects playing the far-end party in the automatic condition sometimes continued talking during the interruptions because they did not know the interruptions were occurringthe break in the voice path was completely silent for the far-end party.

Concern over this potential problem led to the development of two versions of automatic CIDCW. In one, the break in the voice path was silent. In the other, the far-end subjects heard a tone sequence during the $\mathrm{CIDCW}$ interruptions to indicate that communication was temporarily impossible.

\section{METHOD}

\section{Subjects}

We tested 48 subjects and paid them for participating. Subjects were selected from a database of local residents available for testing. Many of them were friends or relatives of others in the database. To facilitate conversation between subjects, pairs of subjects who knew 
each other were often tested together, but pairs of strangers were also tested.

The subjects ranged in age from teenagers to senior citizens. Of the subjects, 16 had Caller ID (Calling Number Delivery) service at home (some of these subjects also had Call Waiting at home), 16 had Call Waiting but not Caller ID, and 16 had neither service at home. The use of these different groups of subjects allowed an analysis of whether one group is more tolerant of interruptions than are the others. Considering that Call Waiting service intrudes on ongoing conversations, Call Waiting subscribers might be relatively tolerant of interruptions. Subjects who have Caller ID service at home might also be tolerant of interruptions. They may find caller ID information so valuable that they do not mind interruptions that bring them this information.

\section{Procedure}

The subjects were tested in pairs. At the beginning of the study, one subject of each pair was assigned the role of "subscriber," and the other subject was designated the "far-end party." Role assignment was done in such a way that subjects of every type (those with Caller ID service at home, those with Call Waiting at home, and those with neither) participated equally often as subscriber and as far-end party. The subscribers were told how CIDCW works and the nature of the interruptions they would hear, but they were not told how long the interruptions would be or when they would occur. The far-end subjects were not told about CIDCW or the interruptions.

To facilitate comparison of automatic and user-controlled CIDCW, all pairs of subjects were tested on both. Half of the subject pairs received the automatic condition followed by the user-controlled condition, and the other half received user-controlled followed by automatic CIDCW. Each condition consisted of five discrete telephone calls, which were preceded by two practice calls. For the practice calls, the subscriber called the experimenter. For the other telephone calls, the subscriber called the far-end party, who sat in a separate room. A modular switching unit driven by a minicomputer completed the connection between the subjects.

During the calls, subjects were free to talk about anything they wanted to, except for CIDCW. It can be difficult for subjects to maintain a conversation over a long period, particularly if they are strangers; therefore they were given the option of playing a "survival simulation" game to stimulate conversation. For the game, subjects read a short description of a survival scenario in which they were lost in the woods and had to walk to civilization. From a list of $\mathbf{3 0}$ items, the subjects had to decide which 15 they would take with them, and they had to rank order the 15 items. The length of each call was determined beforehand by the experimenter and ranged from 2 to $5 \mathrm{~min}$.

The experimenter introduced one interruption into every conversation. The approximate time of the interruption was determined beforehand. For half the subject pairs, the experimenter tried to introduce the interruptions when the subscriber was talking, and for the other half, the interruptions were introduced when the far-end party was talking. The sequence of events experienced by the subscribers during the automatic and user-controlled interruptions is shown in Figure 1.

When an interruption occurred in automatic CIDCW, the switching unit transmitted a $440-\mathrm{Hz}$ tone (i.e., the tone used for Call Waiting service) to the subscriber's line for $0.3 \mathrm{~s}$, followed by a silent break in the voice path for $0.8 \mathrm{~s}$, at the end of which a name and a telephone number appeared on a computer monitor serving as the subscriber's Caller ID CPE. While the subscriber experienced the tone and silent break, half of the far-end subjects were always presented with a silent break for $1.1 \mathrm{~s}$, and half were always presented with a tone for $1.1 \mathrm{~s}$.

It was important for the tone presented to the far-end subjects not to resemble the Call Waiting tone or any other common network signal, such as a dial tone or busy tone, given that the far-end subjects could have believed mistakenly 
that they were receiving a new call or that the telephone connection had been lost. This requirement essentially ruled out the use of a steady or intermittent tone of single or dual frequency. For this reason, a tone sequence was used. The tone heard by the far-end subjects alternated in frequency between 400 and $800 \mathrm{~Hz}$ every $0.3 \mathrm{~s}$.

During the 1.1-s interval, communication between the subscriber and the far-end party was not possible. Immediately after the interval, the connection between the subscriber and the farend party was restored. The subscriber then flashed the switch hook to engage in a conversation with the experimenter, then flashed again to continue the earlier conversation.

In user-controlled CIDCW, the subscriber heard a $440-\mathrm{Hz}$ tone for $0.3 \mathrm{~s}$, while the far-end party received a silent break for $0.3 \mathrm{~s}$. Whenever ready, the subscriber pressed a special button on the face of the telephone marked Caller ID. The button press caused a silent break of $0.7 \mathrm{~s}$ to be presented to both the subscriber and the far-end party, at the end of which a name and a telephone number appeared on the computer monitor on the subscriber's desk. (The silent break was shorter in the user-controlled condition than in the automatic condition because the automatic condition could require extra processing time for technical reasons.) After the silent break, the connection between the subjects was restored. By flashing the switch hook, the subscriber engaged in a conversation with the experimenter, then flashed again to continue the earlier conversation.

After the five telephone calls of a condition (automatic or user-controlled) were completed, the subjects made an assessment of that condition. After both conditions ended, the subjects directly compared automatic and usercontrolled CIDCW. The assessments were made using seven-point scales with labeled end points. In addition to the subjective measures, the experimenter noted any errors that were made in the use of CIDCW. The length of time it took for subscribers to answer new calls was also measured.

\section{RESULTS}

The data were examined initially using analyses of variance with one within-subjects factor (condition: automatic and user-controlled) and three between-subjects factors (type of telephone service at home: Caller ID, Call Waiting,

\section{Automatic}

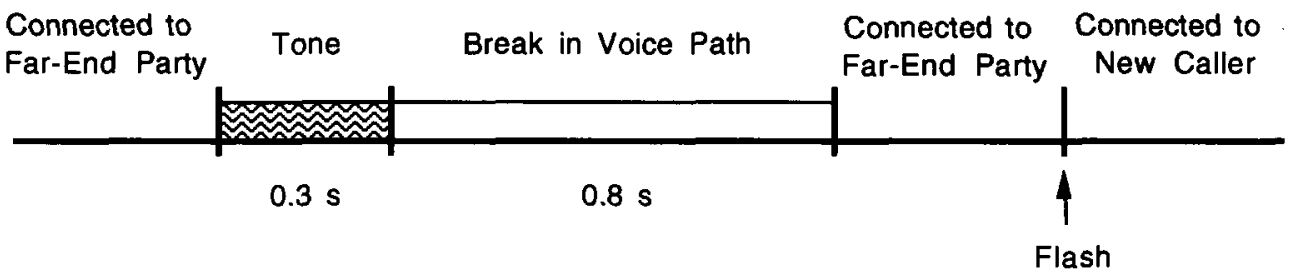

\section{User-Controlled}

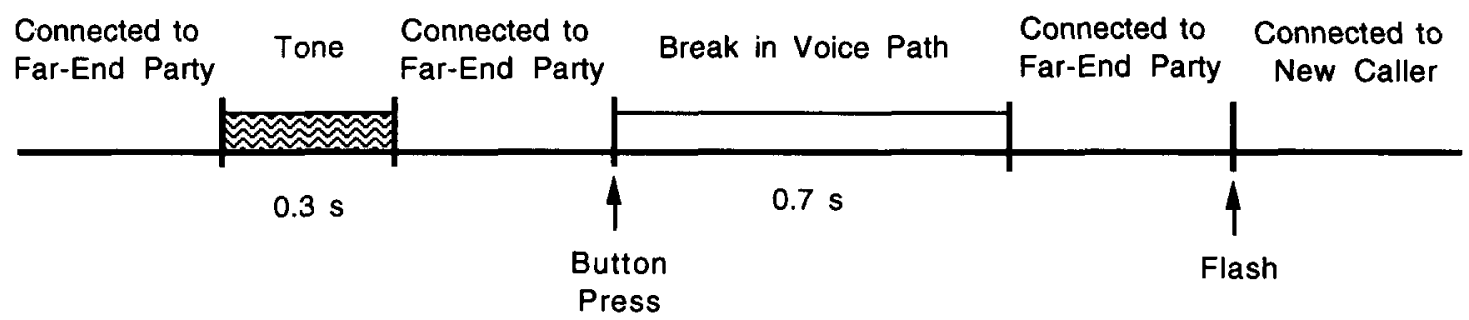

Figure 1. Sequence of events in the automatic and user-controlled interruptions from the subscribers' perspective. At the end of the break in the voice path, subscribers saw the name and telephone number of the new caller. 
or neither; order of the experimental conditions: automatic first or user-controlled first; and which subject was talking when the interruptions occurred: the subscriber or the far-end party). Separate analyses were computed for the subscribers' data and for the far-end subjects' data. Because none of the between-subjects factors was involved in any significant main effects or interactions in any of the analyses, the data were collapsed over these factors and $t$ tests were performed examining differences between the automatic and the user-controlled conditions.

\section{Subscribers}

Subjective ratings. After each condition (automatic and user-controlled), subjects were asked to assess that condition. Although the subjects assessed each condition separately, they were asked the same questions for both conditions, allowing comparisons to be made. Figure 2 lists the questions that were asked, the end-point labels of the seven-point rating scales, and the mean ratings for the automatic and usercontrolled conditions. From the figure it is clear that both forms of CIDCW received excellent ratings, whether the subjects were considering that form of the service alone or comparing it with Call Waiting service. There was no statistically significant difference between the automatic and the user-controlled conditions for any of the questions.

After completing both conditions, the subjects were asked to make direct comparisons of automatic and user-controlled CIDCW. Figure 3 lists the questions that were asked and the mean ratings. The rating scales used user-controlled and automatic as end points. The middle point of the scale, the number 4 , indicates that usercontrolled and automatic were rated equally, numbers less than 4 point toward usercontrolled, and numbers above 4 point toward automatic. (The scales presented to the subjects actually had end points that referred to "the interruptions in the first half of the experiment" versus "the interruptions in the second half."

1. Considering the information that you received (calling number and name), were the interruptions you heard acceptable to you? $(1$ = Completely acceptable, 7 = Completely unacceptable)

2. How much harder was it to use CIDCW than it is to use Call Waiting service? ( $1=$ Not harder, $7=$ Much harder)

3. How much more disruptive to your telephone conversations were these interruptions compared with the interruptions in Call Waiting service? $(1=$ Not more disruptive, 7 = Much more disruptive)

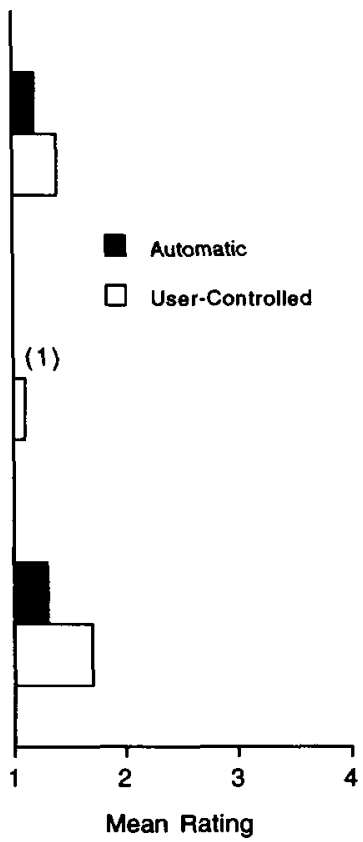

Figure 2. Mean ratings for subscribers when the automatic and user-controlled conditions were judged separately. 
For purposes of analysis, the subjects' ratings were transformed so that the number 1 referred to user-controlled and 7 to automatic.)

For analysis, the ratings were compared with the neutral point of 4 . Statistically significant results were revealed for Questions 2 and 3, respectively, $t(23)=3.5, p=0.002 ; t(23)=2.4$, $p=0.025$. There was a trend toward significance for Question $1, t(23)=1.9, p=0.070$. Automatic CIDCW was rated as being easier to use than user-controlled CIDCW, and it was preferred over user-controlled CIDCW.

When the subjects were directly asked which form of CIDCW they preferred, 18 selected automatic and 6 selected user-controlled. The 3 to 1 margin in favor of the automatic form was significant on a binomial test, $p=0.023$. The subjects also rated the strength of their preference on a seven-point scale. The mean rating given by those who had selected the automatic form was 5.5 compared with 5.7 for those who had selected user-controlled, showing that the subscribers generally felt strongly about their selection. When asked the reason for their choice, those who had selected automatic usually mentioned that it was a simple, straightforward method for finding out who was calling, as it did not require any special actions. Those who had selected user-controlled said that they liked having control over if and when the data would be transmitted.

Observations on how the user-controlled form was used. Full use of the user-controlled form of CIDCW requires that subscribers initiate two breaks into their conversations - one to find out the name and telephone number of the new caller and the second to talk to the new caller. Telephone courtesy demands that subscribers inform the far-end party that they are being put on hold, but in user-controlled CIDCW, because there are two breaks, subscribers can handle the interruptions in three different ways. Before pressing the button to find out who is calling subscribers could excuse themselves, then, if they want to talk to the new caller, flash the switch hook without saying anything. Alternatively, subscribers could press the button to find out who is calling without saying anything but excuse themselves before flashing the switch hook to talk to the new caller. Finally, subscribers could excuse themselves both before pressing the button and before flashing the switch hook.

Although the subjects participated in five telephone calls in the user-controlled condition, all but one of the subscribers adopted one primary

1. Which type of interruption disrupted your telephone conversation more?

2. In which condition was it easier to get the telephone number of the new caller?

3. Which type of interruption do you prefer?

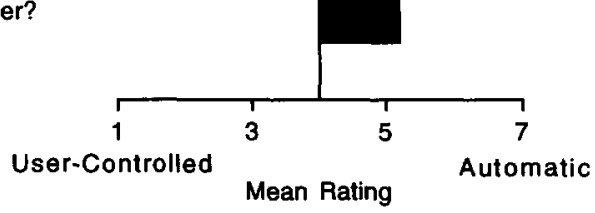

Figure 3. Mean ratings for subscribers when the automatic and user-controlled conditions were compared. 
strategy for handling the interruptions. Of the 23 subscribers with a consistent strategy, 14 excused themselves before pressing the button to find out who was calling, then, without saying anything, flashed the switch hook to talk to the new caller. Four subscribers pressed the button without saying anything, then excused themselves before flashing. Only five subscribers excused themselves twice. Thus most of the subscribers effectively adopted a strategy that increased the length of the user-controlled interruptions-the interruptions lasted from the button press to receive caller ID data, through the switch-hook flash to talk to the new caller, until the switch hook was flashed a second time and communication with the far-end party commenced again.

It should be noted that this strategy might not be a practical way to handle the interruptions if user-controlled CIDCW were actually deployed. In realistic situations, background noise is often present, which the far-end party would hear after the subscribers pressed the button but before they flashed the switch hook to talk to the new caller. If the far-end party heard background noise during this interval, he or she might say something to the subscriber, who would then have to make his or her excuse before flashing. If the subscriber did not say something before flashing in these situations, then the far-end party might think the subscriber had hung up.

Subscriber errors. It was observed that the subscribers made two different types of errors in using CIDCW, both of which occurred in the user-controlled condition. In the user-controlled condition, subscribers had to take two different actions: They pressed a button to find out who was calling, and they flashed the switch hook to talk to the new caller. The first type of error occurred because the subscribers sometimes acted incorrectly. Occasionally they pressed the button when they should have flashed the switch hook, and sometimes, they flashed the switch hook when they should have pressed the button. This type of error was made at least once by 7 of the 24 subscribers, and it affected 8 of the 120 calls. Commission of this type of error means that subscribers might sometimes be connected to a new caller when they only wanted to find out who the new caller was.

The other type of error came from the subscribers' being confused about who they were connected to after they pressed the button to find out who was calling. In Call Waiting service and in automatic CIDCW, the one action subscribers can take (flashing the switch hook) switches them between the far-end party and the new caller. In user-controlled CIDCW, however, the button press makes the name and telephone number of new callers appear on the CPE, but it does not connect subscribers to new callers. Nevertheless, in at least one call 9 of the 24 subscribers erroneously believed that they were connected to the new caller after they pressed the button. This mistake affected 12 of the 120 calls. Confusion over who a person is talking to has the potential for leading to embarrassing situations.

In the automatic condition, the name and telephone number of the new caller were sent automatically. Thus the subscribers had to perform only one action: flashing the switch hook. None of them made any errors in the automatic condition.

Length of time to take the new call. The length of time it took for subscribers to answer the new call was measured for every conversation. These measurements were made from the end of the Call Waiting tone to the moment the subscribers flashed the switch hook to talk to the new caller. The mean time for the automatic condition was $4.8 \mathrm{~s}$ compared with $8.8 \mathrm{~s}$ for the user-controlled condition. The subscribers answered the new call significantly faster in the automatic condition, $t(23)=4.1, p<0.001$.

The longer time for the user-controlled condition could have been attributable either to the necessity of pressing a button to find out who was calling or to the strategy some subscribers adopted of formally excusing themselves from the far-end party before finding out who was calling and excusing themselves again before taking the new call, thus delaying answering the new call. To examine this issue, the time to an- 
swer the call in the user-controlled condition was analyzed as a function of the strategy the subscribers had adopted. The 18 subscribers who excused themselves only once took a mean of $8.5 \mathrm{~s}$ to answer the new call, compared with $10.6 \mathrm{~s}$ for the 5 subscribers who excused themselves twice. Therefore, although making two excuses did delay answering the new call, this factor alone cannot account for the longer time the subscribers took to answer the new call in the user-controlled condition compared with the automatic condition. Apparently the necessity of pressing a button to find out who was calling was involved.

The extra time to answer a new call in the user-controlled condition amounted to $4 \mathrm{~s}$, or a fraction of a ring cycle, which is $6 \mathrm{~s}$. Such a small difference probably would not have any practical consequence in most cases. However, it should be recognized that subjects often react more quickly in laboratory settings than they do in real life. With the increasing use of voice mail, then, it would be more likely with usercontrolled CIDCW than with automatic that new calls subscribers wanted to take would be forwarded to voice mail before they could be answered.

\section{Far-End Subjects}

All of the far-end subjects participated in the user-controlled condition and in one of the two versions of the automatic condition. Half of the far-end subjects were in the automatic with silence condition; during the automatic interruptions, while the subscribers heard a tone followed by silence, they experienced a silent break for $1.1 \mathrm{~s}$. The other half of the far-end subjects participated in the automatic with tone condition; they heard a tone sequence for $1.1 \mathrm{~s}$.

Automatic with silence vs, user-controlled. After each condition (automatic and user-controlled), the far-end subjects were asked to rate that condition. The same questions were asked after both conditions so that comparisons could be made. Figure 4 lists the questions that were asked, the end points of the rating scales, and the mean ratings for the automatic with silence and the user-controlled conditions (left graph). From the figure it is clear that both conditions received good scores. There was no statistically significant difference between the automatic with silence and the user-controlled conditions for either question.

After completing both conditions, subjects were asked to make direct comparisons of automatic with silence and user-controlled CIDCW. The mean ratings are shown in Figure 5 (left graph). The rating scales used user-controlled and automatic as end points. The middle point of the scale, the number 4 , indicates that usercontrolled and automatic were rated equally, numbers less than 4 point toward usercontrolled, and numbers above 4 point toward automatic. The ratings were compared with the neutral point of 4 . Statistically significant results were revealed for both questions: Question $1, t(11)=2.4, p=0.036$; Question 2, $t(11)=3.3$, $p=0.007$. Automatic with silence CIDCW was less disruptive than user-controlled CIDCW, and it was preferred over user-controlled CIDCW.

When the subjects were directly asked which form of CIDCW they preferred, 8 selected automatic, 3 selected user-controlled, and 1 insisted that they were equally acceptable. This pattern of preference was not significant on a binomial test. However, when the subjects rated the strength of their preference using a seven-point scale, those who had preferred the usercontrolled form did not seem to feel as strongly about their selection as those who had preferred automatic with silence; user-controlled received a mean rating of only 1.7 versus 4.4 for automatic CIDCW.

Automatic with tone vs, user-controlled. The far-end subjects who were tested in the automatic with tone condition and the usercontrolled condition also rated each condition. Their mean ratings are shown in Figure 4 (right graph). Highly significant differences were found between the two conditions: Question 1, $t(11)=3.3, p=0.007$; Question $2, t(11)=3.4, p$ $=0.006$. The far-end subjects found the automatic with tone condition unacceptable. 
1. How much more disruptive to your telephone conversations were these interruptions compared with your experiences with Call Waiting service? ( 1 = Not more disruptive; $7=$ Much more disruptive)

2. How much less acceptable were these interruptions compared with those of Call Waiting? (1 = Equally acceptable; 7 = Much less acceptable)
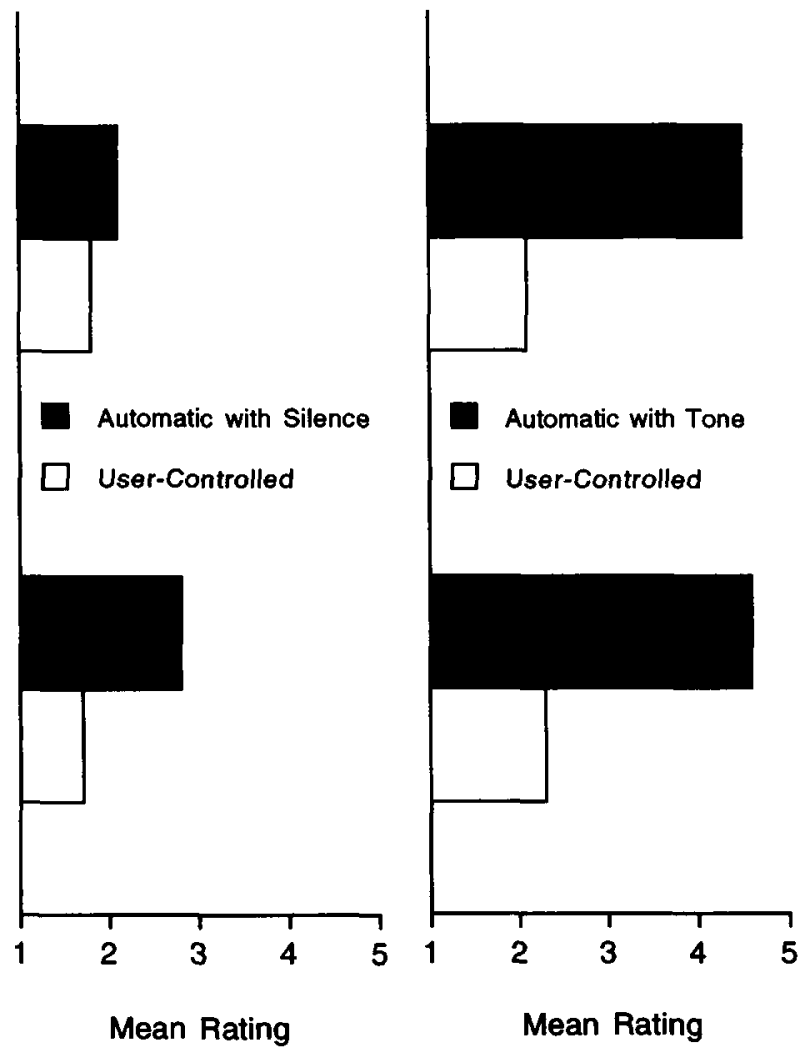

Figure 4. Mean ratings for far-end subjects when the automatic (automatic with silence in left graph, automatic with tone in right graph) and user-controlled conditions were judged separately.

Figure 5 (right graph) shows the far-end subjects' ratings when they made direct comparisons of the automatic with tone and the user-controlled conditions. Compared with the user-controlled condition, the automatic with tone condition was more disruptive-Question $1, t(11)=2.3, p=0.042$ - and less preferred Question 2, $t(11)=3.3, p=0.007$. When asked to choose between the two conditions, 10 far-end subjects selected user-controlled and only 2 selected automatic with tone, $p=0.039$ on a binomial test. All of the subjects felt fairly strongly about their choice.

\section{DISCUSSION}

The purpose of this study was to determine whether it would be preferable to use an automatic or a user-controlled method of briefly interrupting telephone conversations. Accord- ingly, pairs of subjects were tested in the laboratory under conditions that simulated CIDCW. All of the subjects were tested in both automatic and user-controlled conditions, with half of the subjects acting as subscribers to the service and the other half acting as the far-end party. It was found that the subscribers gave both the automatic and the user-controlled conditions excellent scores.

When they made a direct comparison of the two conditions, however, differences were revealed. Compared with the user-controlled condition, the automatic condition was easier to use and was preferred by significantly more subjects. The subscribers preferred automatic over user-controlled by a 3 to 1 margin. Additionally, the subscribers made no errors in the automatic condition, but two different types of errors occurred in the user-controlled condition. One 
1. Which type of interruption disrupted your telephone conversation more?

Automatic with Silence vs. User-Controlled

2. Which type of interruption do you prefer?
Automatic with Tone vs. User-Controlled

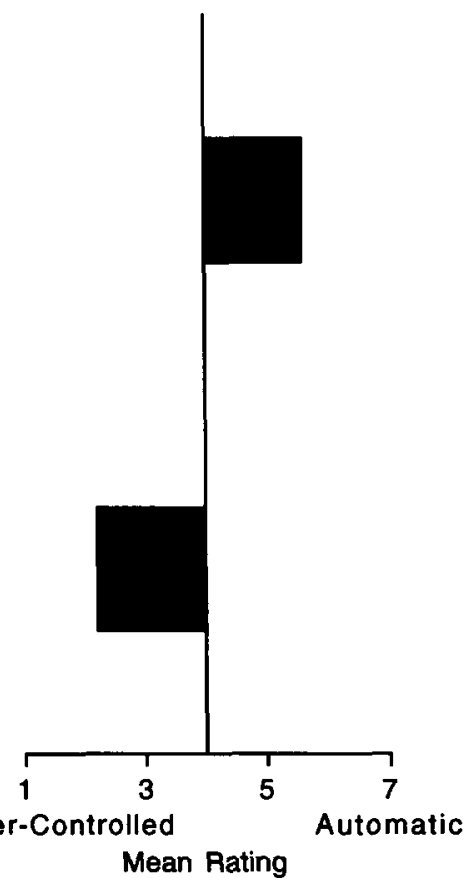

Figure 5. Mean ratings for far-end subjects when the automatic (automatic with silence in left graph, automatic with tone in right graph) and user-controlled conditions were compared.

type of error came from the subscribers' performing the incorrect action. The second type resulted from the subscribers' being confused about the party with whom they were con. nected. Although all of the subjects were able to use user-controlled CIDCW without error after two or three calls, it is clear that user-controlled CIDCW has more potential for confusion than does automatic.

One final difference between automatic and user-controlled CIDCW is that the subscribers were able to answer the new call more quickly in the automatic condition. This means that in automatic CIDCW it would be less likely that the new caller will have hung up or been forwarded to voice mail before subscribers could answer the call.

The results for the subscribers, then, clearly favored an automatic approach over a usercontrolled one. The results for the far-end subjects, however, depended on which version of automatic CIDCW they experienced. The far-end subjects gave both the automatic with silence condition and the user-controlled condition good ratings; however, in direct comparisons, automatic was preferred. When automatic with tone and user-controlled were compared, the results were different. The far-end subjects did not like the automatic with tone condition; they preferred user-controlled CIDCW. The purpose of the tone was to stop the far-end subjects from talking during the interval in which the voice path was broken. Without the tone, in the automatic with silence condition, the far-end subjects continued to speak during this interval because they were unaware that communication was not possible. It is not clear, however, that the far-end subjects in the automatic with tone condition ever came to understand the purpose of the tone. The far-end subjects generally believed that the subscribers simply had Call Waiting service, and the tone served only to 
highlight the fact that something unusual was occurring.

Although there was a clear difference in the subjects' preference for automatic (i.e., automatic with silence) over user-controlled CIDCW, the other experimental manipulations were of little consequence. First, the results did not vary with the kind of telephone service the subjects had at home. It might have been expected that, compared with other types of subjects, those who are accustomed to interruptions during a conversation (i.e., subjects with Call Waiting service at home) might have been more accepting of CIDCW interruptions in general. Also, subjects with Caller ID service might have been more tolerant of CIDCW interruptions because they value receiving caller ID information. This was not the case, however. A prime reason for the nonsignificant difference may have been that all of the subjects seemed to enjoy receiving the name and telephone number of the new caller whether they had Caller ID service at home or not. In addition, all of the subscribers generally found automatic CIDCW to be a better way than user-controlled to find out who was calling.

A second factor that had little consequence concerned who was talking when the CIDCW interruptions occurred. For half of the subject pairs, the interruptions were always introduced when the subscriber was talking, and for the other pairs, the interruptions always occurred when the far-end party was talking. Considering that one of the subjects was always talking when the interruptions began, the interruptions were made as disruptive as possible. (When CIDCW is actually deployed, the interruptions will sometimes occur during natural pauses in the conversation, when no one is talking.)

It might have been expected that subjects would react unfavorably toward the automatic interruptions, particularly when the other party was speaking, because gaps in the other party's discourse would be apparent. This did not turn out to be the case, however. The ratings of the subscribers did not depend on who had been talking because, during the interruptions, they always quickly turned their attention to the Caller ID display to see who was calling, then looked for a place to break the conversation so that they could talk to the new caller. The farend subjects in the automatic with tone condition were always aware of the interruptions regardless of who had been talking. The question arises about how aware the far-end subjects in the automatic with silence condition were of the interruptions. Evidence that addresses this question is available.

After each trial (telephone call) in the experiment, the far-end subjects judged whether they had noticed an interruption using a seven-point scale, from 1 (not noticed) to 7 (very noticed). It is relevant here to examine the ratings given in the automatic with silence condition. When the interruption occurred while the subscriber had been speaking, the mean rating of the far-end subjects was 3.2 ; if the far-end party had been speaking during the interruption, the rating was 1.5. This difference was not significant, $t(10)=$ $1.8, p=0.102$.

The far-end subjects in the automatic with silence condition may not have been fully aware of the interruption, partly because the interruptions sometimes sounded as if they started between syllables, words, or sentences. Interestingly, the far-end subjects often took advantage of the "pause" in the subscribers' discourse to begin talking themselves. This behavior is consistent with psycholinguistic research on the effect of unfilled pauses during conversation (Jefferson, 1989). The far-end subjects occasionally recognized the occurrence of an interruption, but they typically thought it was an ordinary Call Waiting interruption. In any case, the reactions of subjects engaged in natural conversation did not depend on which subject had been talking when the automatic interruptions occurred.

Under different experimental conditions, would the user-controlled form of CIDCW have been favored over the automatic form? For example, in the present study, the subjects were 
engaged in natural conversation, but perhaps subjects would react less favorably toward automatic CIDCW if the other party did all the talking, as this could make gaps in the other party's discourse obvious. Also, in the present study, the subscribers were required to talk with the new caller in every call, and they usually connected with the new caller quickly. Would the results have been different had the subscribers not been required to answer the new call, especially because it was the requirement to talk to the new caller that was involved in the errors made in the user-controlled condition? An advantage CIDCW has over Call Waiting service is that, without having to talk to the new callers, subscribers can acquire enough information to be able to call them back later. Therefore, CIDCW subscribers may elect not to talk to new callers.

Answers to these questions can be found by examining the results of another study of CIDCW. Garberg (1991) also tested subjects in simulations of automatic and user-controlled forms of CIDCW, but the subscribers never answered the new call, though they always attended to the Caller ID display when an interruption occurred. Furthermore, only one subject of each pair did all the talking, while the other subject listened closely. Despite these manipulations, the automatic condition was still preferred by the subscribers by an 11 to 5 margin. The far-end subjects showed equal preference for the two conditions; 8 preferred automatic (i.e., automatic with silence) and 8 preferred user-controlled.

All in all, the question for the subscribers came down to whether they wanted to take action to find out who was calling. Most did not. They preferred the simpler form of CIDCW, the automatic form. Although it is true that usercontrolled CIDCW allows the flexibility of not having to find out who is calling for every new call, undoubtedly most people who subscribe to CIDCW will usually want to find out who is calling. Automatic CIDCW is an easier way to do this. Guidelines for designing the human inter- face for computers (e.g., Apple Computer, 1992) recommend letting the user, rather than the computer, initiate and control actions. A priori it might have been thought that CIDCW interruptions controlled by users would be less intrusive than automatic interruptions, which are relatively long and come at unexpected moments.

This was not found to be the case. In fact, because of the way most subscribers used usercontrolled CIDCW, formally breaking their conversations only once before pressing the button to find out who was calling, the user-controlled interruptions became longer in duration than those of automatic CIDCW. Moreover, the action of pressing the button was found to be intrusive in itself. The principal reason for the subscribers' preference for automatic CIDCW over usercontrolled was that they preferred not to take action to find out who was calling.

Implementing an automatic form of CIDCW raises an issue having to do with the alerting tone. The tone in CIDCW has two purposes. First, it should alert the subscriber to the presence of a new call. Second, it should trigger the subscriber's CPE so that the handset will be muted, preventing the subscriber's speech from corrupting the data and keeping the subscriber from hearing the data transmission. Unfortunately, the CIDCW tone cannot be the $440-\mathrm{Hz}$ tone used for Call Waiting service; detection of that tone by a machine would not be reliable when speech is present because speech commonly has energy of high amplitude at $440 \mathrm{~Hz}$. Extensive human factors (Fusco and Katz, 1992) and signaling (Bellcore, 1993) work has led to the development of a short tone of 2130 and 2750 $\mathrm{Hz}$ that can be used to indicate to CPE that the handset should be muted. This new tone is short enough that it can be appended to the $440-\mathrm{Hz}$ tone with which customers of Call Waiting service are familiar.

A second issue has to do with how long an automatic interruption can be and still be acceptable to customers. Preliminary work on this issue (Katz and Fusco, 1993) has suggested that 
under certain circumstances, the interruption may be longer than the approximately $1 \mathrm{~s}$ tested in the present study. Nevertheless, the importance of keeping unexpected interruptions as short as possible should be emphasized.

It can be difficult to know from a short-term study what users' attitudes would be after longterm exposure to a service. Over the long term, the novelty of getting caller ID information might diminish, and user satisfaction with unexpected, automatic interruptions might decline. However, if customers want to find out who is calling, automatic CIDCW is definitely a less intrusive way to do so than Call Waiting service, which requires that customers put the far-end party on hold and talk to the new caller to determine who the new caller is.

Moreover, it is not clear that user satisfaction with automatic CIDCW would ever become lower than that of user-controlled CIDCW. After all, even in a short study, subscribers did not like having to press a button to find out who was calling. They might like pressing a button still less over the long term, maintaining a preference for automatic over user-controlled inter. ruptions.

\section{ACKNOWLEDGMENTS}

The work reported here was conducted while the author worked at Bellcore.

\section{REFERENCES}

Apple Computer. (1992). Macintosh human interface guidelines. Reading, MA: Addison-Wesley.
Bellcore. (1989). ISDN access call control switching and signaling requirements (Tech. Reference TR-TSY-000268, Issue 3). Piscataway, NJ: Author.

Bellcore. (1993). A method and apparatus for detecting a dual tone signal in the presence of speech (Special Report SRTSY-002578, Issue 1). Piscataway, NJ: Author.

Cavanaugh, J. R., Hatch, R. W., and Sullivan, J. L. (1976). Models for the subjective effects of loss, noise, and talker echo on telephone connections. Bell System Technical Journal, 55, 1319-1371.

Fusco, M., and Katz, R. B. (1992). Catch a rising tone: Selecting a tone for a new calling service. In Proceedings of the Human Factors Society 36th Annual Meeting (pp. 227-231). Santa Monica, CA: Human Factors and Ergonomics Society.

Garberg, R. (1991). AT\&T's study of Caller ID on Call Waiting. Presented at the Caller ID on Call Waiting Open Vendor Forum, Phoenix, AZ.

Gruber, J. G., and Strawczynski, L. (1985). Subjective effects of variable delay and speech clipping in dynamically managed voice systems. IEEE Transactions on Communications, COM-33, 801-808.

Jefferson, G. (1989). Preliminary notes on a possible metric which provides for a "standard maximum" silence of approximately one second in conversation. In D. Roger and P. Bull (Eds.), Conversation. Philadelphia: Multilingual Matters.

Katz, R. B., and Fusco, M. (1993). On interrupting telephone calls: The effect of interruption length. In Proceedings of the 14th International Symposium on Human Factors in Telecommunications (pp. 101-107). Heidelberg, Germany: R. v. Decker.

Klemmer, E. T. (1967). Subjective evaluation of transmission delay in telephone conversations. Bell System Technical Joumal, 46, 1141-1147.

Miller, G. A., and Licklider, J. C. R. (1950). The intelligibility of interrupted speech. Joumal of the Acoustical Society of America, 22, 167-173.

Schilling, D. L., Pickholtz, R. L., and Milstein, L. B. (1990) Spread spectrum goes commercial. IEEE Spectrum, 27(8), $40-41,44-45$.

Date received: November 30,1993

Date accepted: February 4, 1995 